\title{
A Proposed Two-Stage Two-Tether Scientific Mission at Jupiter
}

\author{
Mario Charro, Juan R. Sanmartin, Claudio Bombardelli, Antonio Sanchez-Torres, \\ Enrico C. Lorenzini, Henry B. Garrett, and Robin W. Evans
}

\begin{abstract}
A two-stage mission to place a spacecraft (SC) below the Jovian radiation belts, using a spinning bare tether with plasma contactors at both ends to provide propulsion and power, is proposed. Capture by Lorentz drag on the tether, at the periapsis of a barely hyperbolic equatorial orbit, is followed by a sequence of orbits at near-constant periapsis, drag finally bringing the SC down to a circular orbit below the halo ring. Although increasing both tether heating and bowing, retrograde motion can substantially reduce accumulated dose as compared with prograde motion, at equal tether-to-SC mass ratio. In the second stage, the tether is cut to a segment one order of magnitude smaller, with a single plasma contactor, making the SC to slowly spiral inward over several months while generating large onboard power, which would allow multiple scientific applications, including in situ study of Jovian grains, auroral sounding of upper atmosphere, and space- and time-resolved observations of surface and subsurface.
\end{abstract}

\section{INTRODUCTION}

$\mathbf{P}$ OWER AND propulsion needs have been critical issues in missions to the outer planets. In all past missions to Jupiter in particular, instruments onboard were powered by radioisotope thermal (or power) generators (RTGs), which present a number of problems. Furthermore, a recent report from its National Research Council warned that the U.S. was running out of RTG fuel (Pu-238); production was stopped over 20 years ago, although the National Aeronautics and Space Administration (NASA) continues to acquire about $5 \mathrm{~kg}$ each year from Russia [1]. The pending Juno mission to Jupiter chose to use solar power, which is possible due to its limited mission duration of one year, a polar Jovian orbit that keeps its solar panels in constant sunlight and limits its radiation exposure, and an energy-efficient operation plan. Also, the twospacecraft (SC) Europa Jupiter System Mission, which was tentatively planned by NASA and ESA for 2020, was to have used 540 W from RTGs in NASA's Jupiter Europa Orbiter (JEO) and $51-\mathrm{m}^{2}$ solar panels in ESA's Jupiter Ganymede Orbiter (JGO).

In turn, capture into and touring in the deep Jovian gravitational well typically require a very high wet-mass fraction if chemical rockets (in addition to gravity-assist operations) are used for propulsion, as was always the case in the past. In particular, the JEO system mass budget required $2646 \mathrm{~kg}$ of propellant and $1490 \mathrm{~kg}$ of flight system mass and launch vehicle adapter, with $973 \mathrm{~kg}$ left for contingency and margin, for a launch mass of $5040 \mathrm{~kg}$. Corresponding values for the JGO system are 2562, 1147, 653, and $4362 \mathrm{~kg}$, which is only 1.7 times propellant mass, respectively. Both JEO and JGO were to have used ballistic trajectories with gravity assists from Venus and Earth [2].

Beyond a range of other applications [3], bare electrodynamic tethers can provide both power and propulsion, with just tether hardware accounting for tether subsystem mass. Basically, a planetary magnetic field induces a motional electric field $\boldsymbol{E}_{m}$ in the orbiting-tether frame and exerts a Lorentz force on the current that $\boldsymbol{E}_{m}$ drives through the tether. If used at Jupiter, tethers with a small tether-hardware/SC mass ratio $m_{t} / M_{\mathrm{SC}}$ have been shown able to perform Jovian orbit insertion, followed by a moon tour with near-zero wet mass, while providing power throughout the entire operation [4], [5]. A total of 40 flybys of Ganymede, Europa, and Io (as against 25 moon flybys planned for JEO) could be carried out before some limiting radiation dose did accumulate.

Radiation dose is the main constraint on tether use at Jupiter because operation is quite requiring ambient conditions, i.e., plasma density and magnetic field, so orbits must reach near Jupiter (the Io torus density is similarly high, but the magnetic field is weaker). For missions such that orbits skip the Jovian radiation belts, anyway, tethers would be greatly useful. The Juno case is a particular example. It has been recently shown that a moderately light tether might provide the power that its $S C$ requires [6].

In this paper, we consider a two-stage two-tether mission. Following capture as described in [4], the SC tether would have its apoapsis progressively lowered to finally reach a circular orbit at the periapsis of the capture orbit, about 1.3-1.4 times the Jovian radius $R_{J}$, skipping moon flybys as considered in [5] to reduce dose accumulation. In the second stage, a short segment of the original tether makes its SC to slowly spiral inward, in a controlled manner, keeping below the belts throughout while generating power on board for science applications, for which 


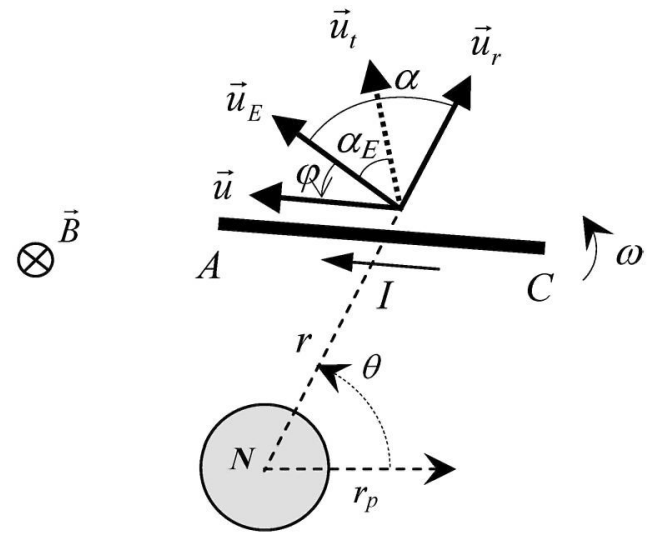

Fig. 1. Relative positions of unit vectors for motional electric field and spinning tether; $\boldsymbol{A}$ and $\boldsymbol{C}$ are the anodic and cathodic ends, respectively, and $u_{E}$ is the unit vector along the motional field $\boldsymbol{E}_{m}$, which is perpendicular to the relative velocity $\boldsymbol{v}^{\prime}\left(\alpha_{E}\right.$ different from $\left.\pi / 2\right)$. For high tether spin rate, angle $\varphi$ would range over $360^{\circ}$ at near-constant orbit position.

the proximity to Jupiter, under the evolving in situ conditions surrounding the SC, offers a world of opportunities.

Section II deals with evolution through highly elliptical orbits to an initial circular equatorial orbit below the belts. Accumulated dose and tether heating and bowing issues are discussed in Section III, comparing retrograde and prograde orbit performances. In Section IV, evolution of orbits below the belts, keeping them near circular throughout, and the power generated on board are considered. Scientific applications are discussed in Section V. Conclusions are presented in Section VI.

\section{REACHING Down to A LOW JOVIAN ORBIT}

Lowering apojove after capture typically requires a large number of orbits. As the eccentricity decrement per orbit will be small, calculations are carried out here as if eccentricity $e$, although different from unity, is kept constant over each orbit. Also, perijove radius $r_{p}$ is taken as constant throughout because drag becomes rapidly small away from perijove. The energy per unit mass and the eccentricity decrement for each elliptical orbit can then be written as

$$
\varepsilon=\frac{-\mu_{J}}{2 r_{p}}(1-e) \rightarrow \Delta e=\frac{2 r_{p}}{\mu_{J}} \Delta \varepsilon=\frac{2 r_{p}}{\mu_{J}} \frac{W_{\mathrm{drag}}}{M_{\mathrm{SC}}}
$$

where $W_{\text {drag }}$ is the work per orbit by the Lorentz drag $\vec{F}_{m a g}$. Drag power reads

$$
\begin{aligned}
\dot{W}_{\mathrm{drag}} & =\vec{\nu} \cdot \vec{F}_{\mathrm{mag}}=\nu \vec{u}_{t} \cdot\left(-I_{\mathrm{av}} \vec{u} \times L B \vec{k}\right) \\
& =-L B \nu I_{\mathrm{av}} \sin \left(\varphi+\alpha_{E}\right)
\end{aligned}
$$

where $\vec{\nu}$ is the SC velocity, $I_{\mathrm{av}}$ is the tether current averaged over its length $L$, and $B$ is the Jovian magnetic field. Fig. 1 shows the directions of unit vectors and angles. Note that, since orbits are equatorial, the no-tilt magnetic field (assumed here) is perpendicular to both SC and corotating plasma velocities.

Both hollow-cathode contact impedance and radiation impedance for current closure in the Jovian plasma are negligible [4]. With ohmic effects being typically weak and assuming relatively low use of power on board, the tether, if bare of insulation [7], collects electrons over near its entire length. Since low density $N_{e}$ and high temperature $T_{e}$ imply a large Debye length in the Jovian plasma, current $I_{\mathrm{av}}$ is given by the orbital-motion-limited law

$$
I_{\mathrm{av}}=\frac{2}{5} \frac{2 w L}{\pi} e N_{e} \sqrt{\frac{2 e E_{t} L}{m_{e}}}
$$

where $w$ is the width of the bare tether. The tether is shaped as a thin tape because this geometry has the lowest cross-sectional area for a given collecting perimeter. Also, $e$ and $m_{e}$ are the electron charge and mass, respectively, $E_{t}$ is the motional-field vector projection along the tether (Fig. 1)

$$
E_{t}=\vec{u} \cdot \vec{E}_{m}=E_{m} \cos \varphi, \quad \vec{E}_{m}=\vec{\nu}^{\prime} \times \vec{B}, \quad\left(E_{m}=\nu^{\prime} B\right)
$$

and $\vec{\nu}^{\prime}=\nu \vec{u}_{t} \pm \Omega_{J} r \vec{u}_{\theta}$ is the SC-to-plasma relative velocity, with $\Omega_{J}$ as the spin rate of Jupiter. Minus/plus signs correspond to prograde/retrograde orbits, respectively.

The tether would spin in the equatorial orbital plane, perpendicular to the magnetic field, with hollow cathodes at both ends taking active turns as each end becomes cathodic. For a highenough spin rate $\omega_{t}$, centrifugal forces will keep it straight, and the $\varphi$-averaged drag power at near-constant orbit position takes the form

$$
\begin{aligned}
\left\langle\left|\dot{W}_{\mathrm{drag}}\right|\right\rangle_{\varphi} & =C^{*} L B \frac{4 w L}{5 \pi} e N_{e} \sqrt{\frac{2 e L B}{m_{e}}} \frac{\nu \nu^{\prime} \sin \alpha_{E}}{\left(\nu^{\prime 2}\right)^{1 / 4}} \\
C^{*} & =\left\langle\cos ^{3 / 2} \varphi\right\rangle \approx 0.556 .
\end{aligned}
$$

The tethered system can be spun up by using chemical thrusters at the tip masses with angular momentum staying constant once the final speed is attained. After assuming the parameters in [5] with a final spin period of 12 min, a tether length of $50 \mathrm{~km}$, and an overall system mass of either 600 or $1000 \mathrm{~kg}$, spinning can be achieved with about 40 or $77 \mathrm{~kg}$ of hydrazine, respectively. As the spin-up time depends on thrust level, 21 and $39 \mathrm{~min}$ will be required for the two options assuming a thrust level of $40 \mathrm{~N}$. For previous studies on alternative spinning techniques of tethered systems, see [8] and [9].

Using the condition $\vec{E}_{m} \perp \vec{\nu}^{\prime}$ and conservation of angular momentum $r \vec{\nu} \cdot \vec{u}_{\theta}=r_{p} \nu_{p}$, along with eccentricity during each orbit, yields

$$
\begin{aligned}
\nu^{\prime 2} & =\nu^{2}+\Omega_{J}^{2} r^{2} \pm 2 \Omega_{J} r_{p} \nu_{p} \\
\nu \nu^{\prime} \sin \alpha_{E} & =\nu \vec{u}_{t} \cdot\left(\nu \vec{u}_{t} \pm \Omega_{J} r \vec{u}_{\theta}\right)=\nu^{2} \pm \Omega_{J} r_{p} \nu_{p}
\end{aligned}
$$

with $\nu_{p}$ as the SC velocity at perijove and $\nu^{2}$ given by the vis viva equation

$$
\nu^{2}=\frac{\mu_{J}}{r_{p}}\left(\frac{2 r_{p}}{r}-1+e\right) .
$$

Equations (5)-(7) can be used to show that, as expected, retrograde orbits produce greater drag than prograde orbits for any choice of tether and orbital parameters. 
To obtain drag work over an entire orbit as required in (1), we use (6)-(8) in integrating (5) along the drag part of the orbit

$$
\left|W_{\mathrm{drag}}\right|=2 \int_{r_{p}}^{r_{u}} \frac{\left\langle\left|\dot{W}_{\mathrm{drag}}\right|\right\rangle_{\varphi} d r}{d r / d t} .
$$

The upper limit is the smaller of the apojove radius $r_{a}=$ $r_{p}(1+e) /(1-e)$ and the radius where the tangential relative velocity vanishes, given by condition $\nu^{2} \pm \Omega_{J} r_{p} \nu_{p}=0$; clearly, $r_{u} \equiv r_{a}$ for all retrograde orbits. As previously noticed, the perijove neighborhood makes a dominant contribution to the integral. We use a no-tilt no-offset dipole model of the magnetic field, normalized with its value at the stationary orbit radius $a_{s}=\left(\mu_{J} / \Omega_{J}^{2}\right)^{1 / 3} \approx 2.24 R_{J}$

$$
B / B_{s} \equiv \widetilde{B}=a_{s}^{3} / r^{3}, \quad B_{s} \approx 0.38 \text { gauss. }
$$

The field at the Jovian surface, i.e., $2.24^{3} \times 0.38 \mathrm{G}$, is over one order of magnitude greater than that at the Earth's surface. For $N_{e}$, the Divine-Garrett model of the thermal electron density in the plasmasphere at the equator yields [10]

$$
\begin{aligned}
N_{e} / N_{s} & \equiv \widetilde{N}_{e}=\exp \left(\frac{r_{0}}{r}-\frac{r_{0}}{a_{s}}\right) \\
N_{s} & \approx 1.44 \times 10^{2} \mathrm{~cm}^{-3} \quad r_{0} \approx 7.68 R_{J} .
\end{aligned}
$$

Angular momentum conservation and the orbit equation $1+$ $e \cos \theta=(1+e) r_{p} / r$ are used to determine $d r / d t$

$$
\frac{d r}{d t}=\frac{\nu_{p}}{1+e} \sqrt{e^{2}-\left(\frac{1+e}{\hat{r}}-1\right)^{2}} .
$$

Finally, using (5) and (12) in (9), we find (13) and (14), shown at the bottom of the page, where $\widetilde{r} \equiv r / r_{p}$ and $\Omega_{e s} \equiv$ $e B_{s} / m_{e}$ is the electron gyrofrequency at $a_{s}$.

\section{Tether Performance in Prograde Versus RETROGRADE MOTION}

The decrement $\Delta e$ at fixed $e$ is then given by

$$
\Delta e=-\frac{16 \sqrt{2}}{5 \pi} C^{*} \frac{m_{t}}{M_{\mathrm{SC}}} \frac{m_{e} N_{s}}{\rho_{t}}\left(\frac{\Omega_{e s}}{\Omega_{J}}\right)^{3 / 2} \frac{L^{3 / 2} r_{p}}{h a_{s}^{3 / 2}} \widetilde{W}_{\mathrm{drag}}\left(r_{p}, e\right)
$$

where $m_{t}, h$, and $\rho_{t}$ are the tape mass, thickness, and density, respectively. Fig. 2 shows the $\Delta e$ versus $e$, at $r_{p}=1.3 R_{J}$, for retrograde and prograde motions, for an aluminum tether. For

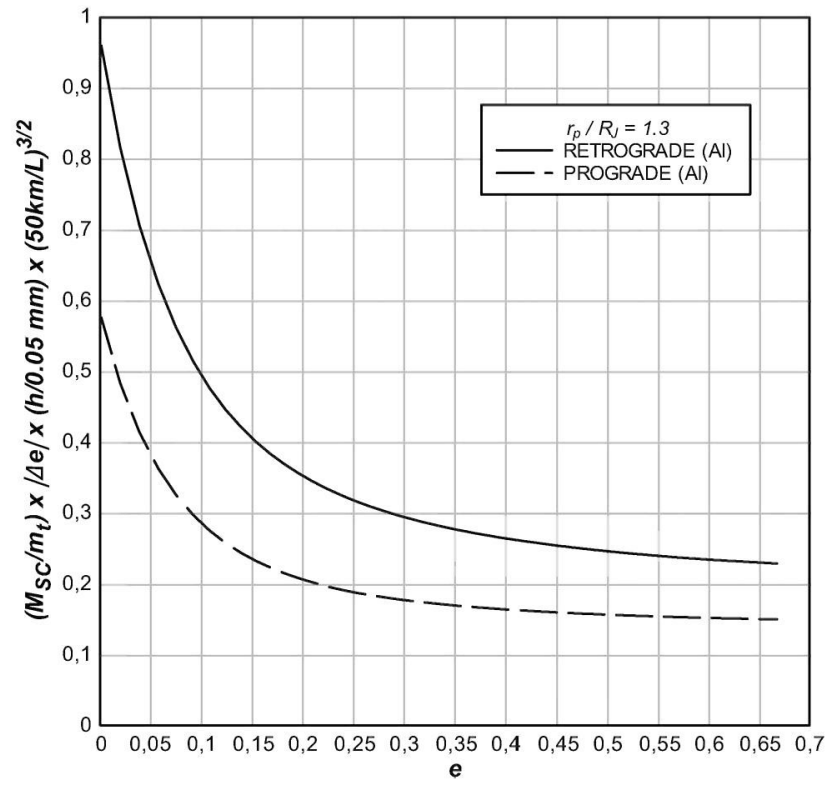

Fig. 2. $\left(M_{\mathrm{SC}} / m_{t}\right) \cdot|\Delta e| \cdot(50 \mathrm{~km} / L)^{3 / 2}$ versus $e$ for $r_{p}=1.3 R_{J}$. Retrograde and prograde cases are shown in solid and broken lines, respectively.

equal tether parameters and SC-to-tether mass ratio, retrograde motion would need fewer orbits to bring the SC down to a circular orbit. For either motion, performance increases with the ratio $L^{3 / 2} / h$, independently of tape width $w$. Tether dimensions $L=$ $50 \mathrm{~km}, w=3 \mathrm{~cm}$, and $h=0.05 \mathrm{~mm}$, for example, yield a mass $m_{t}=202.5 \mathrm{~kg}$, corresponding to $M_{\mathrm{SC}}=600$ and $1000 \mathrm{~kg}$ for $M_{\mathrm{SC}} / m_{t}$ values of about three and five, respectively.

The number of perijove passes is a metric for radiation dose. We use the so-called Galileo interim radiation electron model in the calculations [11]. Fig. 3 shows the dose increment per orbit versus eccentricity for four perijove values, considering an aluminum spherical shield shell of $10-\mathrm{mm}$ thickness for all $4 \pi$ steradians. Note that radiation dose first increases as $e$ is increased and then decreases. Fig. 4 uses calculations for Figs. 2 and 3 to determine how dose accumulates as apojove is lowered, for $r_{p}=1.3 R_{J}$. Fig. 5 shows the radiation dose accumulated, when reaching the circular orbit, versus perijove radius; performance in prograde orbits is worst at the highest $r_{p}$. Dose can be over $60 \%$ higher for prograde motion.

The effect of type of motion on both tether heating and bowing is opposite, however [4]. In the energy balance, which is a local one, ohmic effects and solar heating may be ignored. Furthermore, for a conservative estimate, the tape may be taken as in quasi-steady equilibrium; radiation loss then balances heating from the impact of collected electrons. Maximum

$$
\begin{aligned}
\left|W_{\mathrm{drag}}\right| & =\frac{8 \sqrt{2}}{5 \pi} C^{*} m_{e} N_{s} \Omega_{e s}^{3 / 2} \sqrt{\frac{\mu_{J}}{\Omega_{J}}} w L^{5 / 2} \widetilde{W}_{\mathrm{drag}}\left(r_{p}, e\right) \\
\widetilde{W}_{\mathrm{drag}} & \equiv \int_{1}^{\widetilde{r}_{u}} \frac{\sqrt{\widetilde{r}_{a}} \widetilde{N} \widetilde{N} \widetilde{B}^{3 / 2} d \widetilde{r}}{\sqrt{\widetilde{r}-1} \sqrt{\widetilde{r}_{a}-\widetilde{r}}} \frac{\left[(2 / \widetilde{r})+e-1 \pm(1+e) \Omega_{J} r_{p} / \nu_{p}\right] \Omega_{J} r_{p} / \nu_{p} \sqrt{1+e}}{\left[(2 / \widetilde{r})+e-1 \pm 2(1+e) \Omega_{J} r_{p} / \nu_{p}+(1+e) \widetilde{r}^{2}\left(\Omega_{J} r_{p} / \nu_{p}\right)^{2}\right]}
\end{aligned}
$$




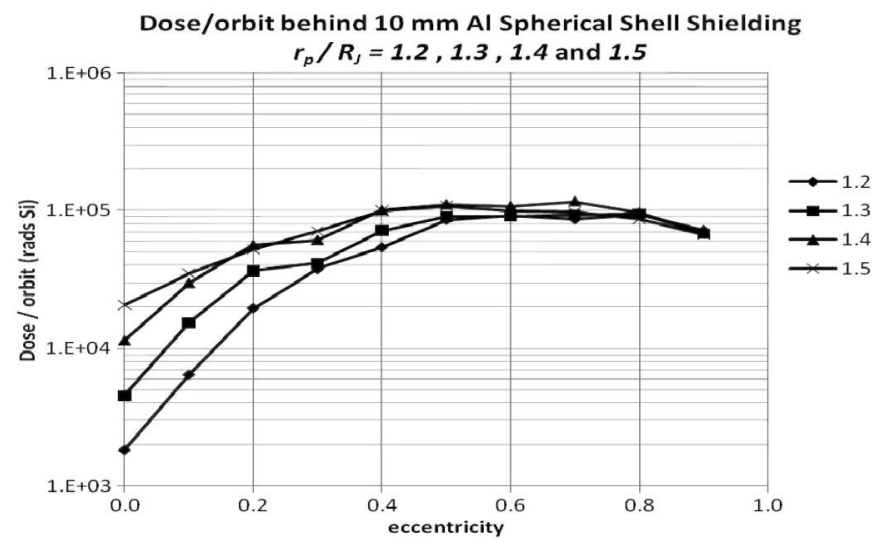

Fig. 3. Dose per orbit versus eccentricity, with an aluminum spherical shell shielding of $10 \mathrm{~mm}$. Four values of perijove radius are considered.

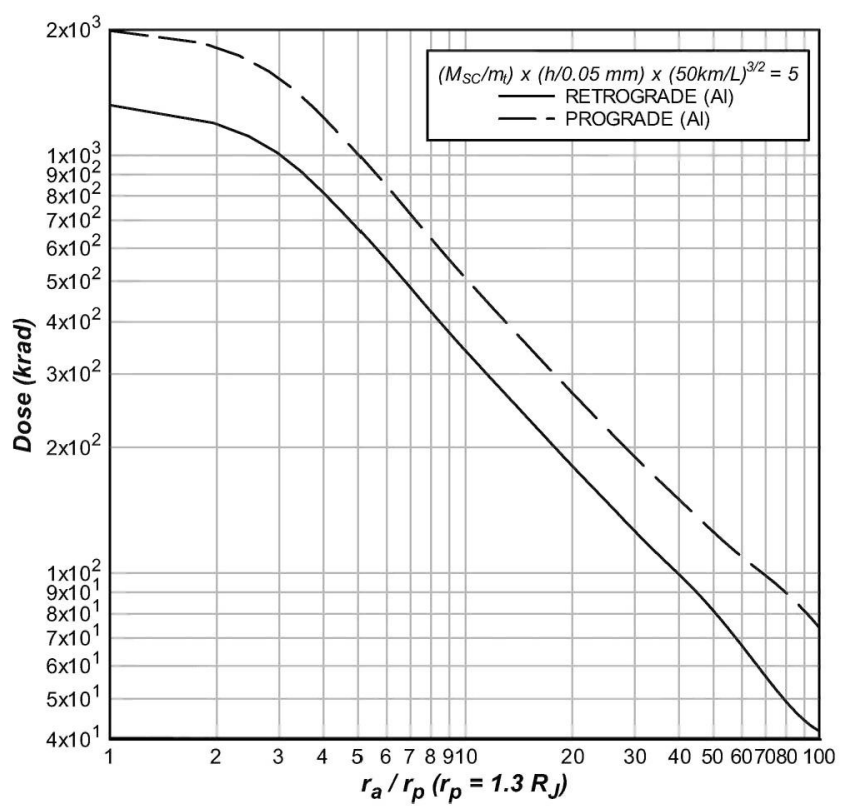

Fig. 4. Radiation dose accumulation from capture to low Jovian orbit for $r_{p} / R_{J}=1.3$ and both retrograde and prograde cases.

temperature occurs around perijove during capture, at each tape end when anodic, and scales with $L^{3 / 8}$

$$
\begin{aligned}
T_{\max }^{4}=\frac{N_{s} m_{e}}{2^{5 / 4} \varepsilon_{t} \pi \sigma_{B}} & \left(\frac{e \nu_{s} B_{s} L}{m_{e}}\right)^{3 / 2} \tilde{r}_{M}^{2} \\
& \quad \times \exp \left(2.72 \widetilde{r}_{M}^{2 / 3}-3.43\right)\left(\widetilde{r}_{M} \pm 1\right)^{3 / 2}
\end{aligned}
$$

where $\varepsilon_{t}$ and $\sigma_{B}$ are the tether emissivity and StefanBoltzmann constant, respectively, and $\nu_{s} \equiv \sqrt{2 \mu_{J} / a_{s}}$ is the parabolic velocity at $a_{s}$, and we introduced $\widetilde{r}_{M} \equiv \sqrt{2}\left(a_{s}\right)$ $\left.r_{p}\right)^{3 / 2}$ [4]. Fig. 6 shows the maximum temperature versus perijove radius for retrograde and prograde orbits, for $\varepsilon_{t}=0.8$.

A simple and conservative estimate of bowing considers the statics of a rope loaded laterally by the Lorentz force $d F / d s=$ $B I(s)$ and supported at the two ends [4]

$$
\frac{d^{2} y}{d s^{2}}=\frac{B I(s)}{T} \Rightarrow \frac{\left|y_{\max }\right|}{L} \propto \frac{L B I_{\mathrm{av}}}{T}
$$

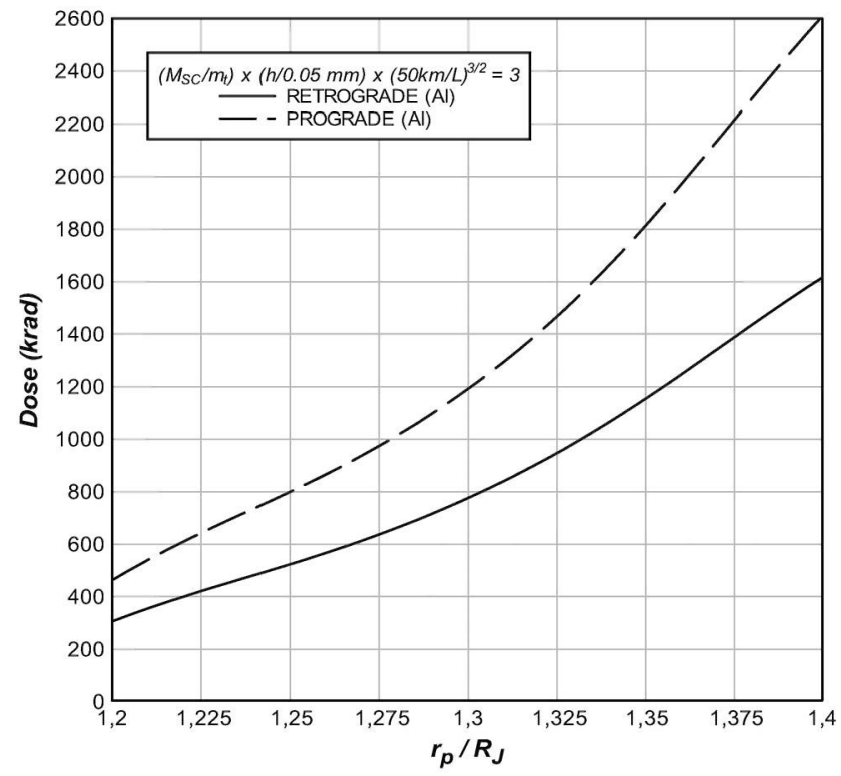

Fig. 5. Accumulated radiation dose down to low Jovian orbit.

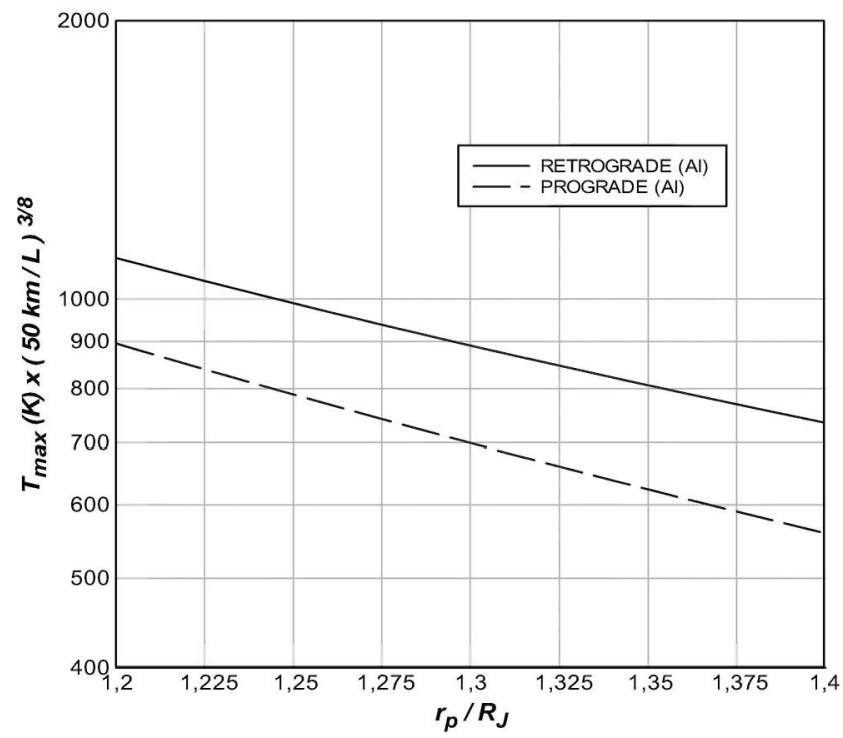

Fig. 6. Maximum temperature versus perijove radius for both retrograde and prograde orbits.

where $y$ is the lateral rope deflection assumed small compared to $L, T$ is the tensile force, and $s$ measures the distance along the tether. For all parameters equal, motional field and, thus, Lorentz force and bowing are greater for retrograde cases. Maximum bowing occurs where Lorentz force is the highest, at a distance $0.56 L$ from the anodic end [3] for $\varphi=0$, at perijove during capture

$$
\begin{aligned}
\frac{\left|y_{\max }\right|}{L}=0.016 \frac{L w}{T} N_{s} e B_{s} L\left(\frac{e \nu_{s} B_{s} L}{m_{e}}\right)^{1 / 2} \widetilde{r}_{M}^{8 / 3} \\
\quad \times \exp \left(2.72 \widetilde{r}_{M}^{2 / 3}-3.43\right)\left(\widetilde{r}_{M} \pm 1\right)^{1 / 2}
\end{aligned}
$$

Fig. 7 shows the maximum bowing versus $r_{p}$, as both proportional to $L^{5 / 2} / h$ and inversely proportional to tensile stress $T /(w h)$. With gravity gradient being negligible, $T /(w h)$ scales 


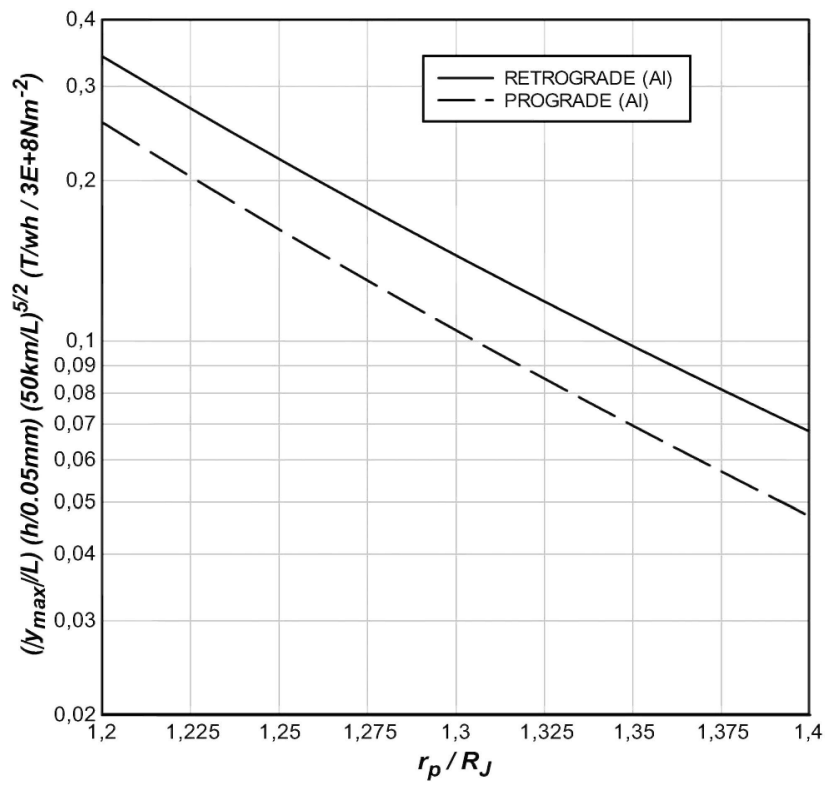

Fig. 7. Maximum bowing versus perijove radius.

with $\rho_{t} \omega^{2} L^{2}$ for fixed $M_{\mathrm{SC}} / m_{t}$. This result is unaffected by the type of motion, making the maximum bowing scale with $\sqrt{ } L /\left(\rho_{t} \omega_{t}^{2} h\right)$. A high spin reduces bowing but could result in too high a tensile stress.

\section{Orbit Evolution Below the Radiation Belts}

In this second stage, at the high $N_{e}$ and $B$ values prevalent below the belts, tether lengths on the order of $50 \mathrm{~km}$, as required for capture and lowering to a low Jovian orbit, would result in a too rapid deorbiting that is inconvenient for science. At this point, the tether is cut, leaving a short segment with just one hollow cathode, to drag the SC down. The cut will produce a recoil of the tether segment that eventually reaches an extended configuration because of the centrifugal forces due to the system spin. The loss of one hollow cathode results in $I_{\text {av }}$ being negligible half the time and a reduced $\varphi$-average, $\left\langle\cos ^{3 / 2} \varphi\right\rangle \approx 0.278$. For slow inward spiraling, the SC will be at any time in a near-circular equatorial orbit, just characterized by its radius $a(t)$.

During each half spin period with the remaining hollow cathode at the cathodic end, the drag power is

$$
\dot{W}_{\mathrm{drag}}=\vec{\nu} \cdot \vec{F}_{\mathrm{mag}}=-L B(a) \nu(a) I_{\mathrm{av}}(a) \cos \varphi .
$$

Since orbit and plasma velocities are now parallel, $\mathbf{E}_{\mathbf{m}}$ is aligned with the local vertical, making $\alpha_{E}=\pi / 2$ in (2). The tether would also be generating power, for science applications, at some useful load of impedance $Z_{\text {load }}$ characterized by the point where the tether bias vanishes, i.e., by the length $\zeta L(\zeta<$ 1 ) of the segment at positive bias (Fig. 8). The bare tether would collect electrons over that anodic segment, with $\zeta=1$ corresponding to no load. Ion current to the cathodic segment is negligible. We then have [3], [7]

$$
I_{\mathrm{av}}=\frac{2}{5} \frac{2 w L}{\pi} e N_{e}(a) \sqrt{\frac{2 e E_{m}(a) L \cos \varphi}{m_{e}}} \varsigma^{3 / 2} \frac{5-2 \varsigma}{3} .
$$

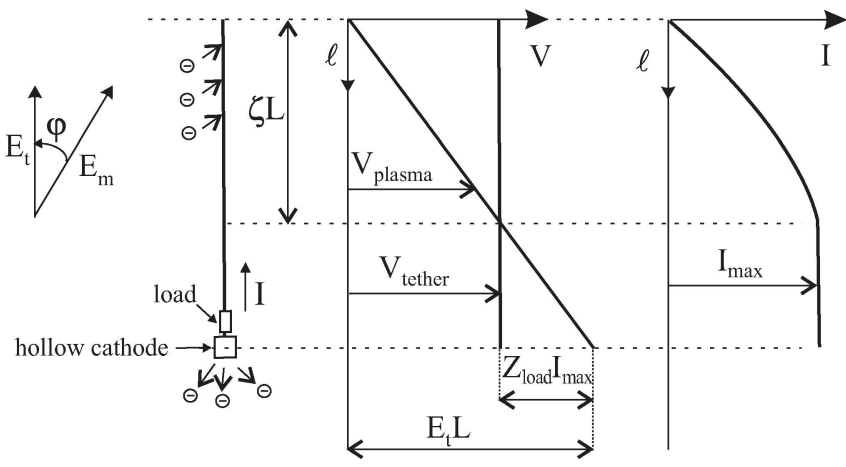

Fig. 8. Schematics of the short bare tether in generator mode with negligible ohmic losses (straight tether-potential line), during each half spin period with the hollow cathode at the cathodic end, throughout the second-stage deorbiting.

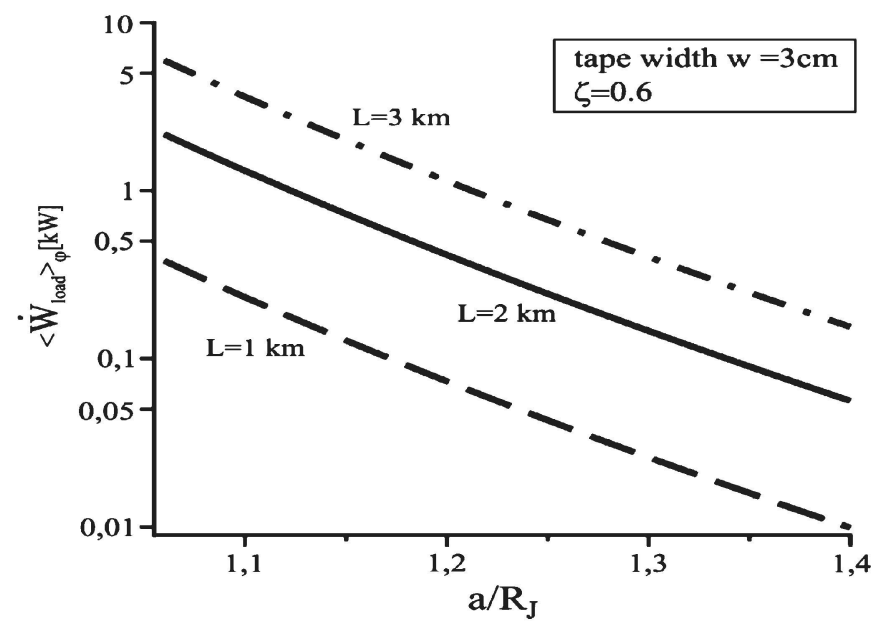

Fig. 9. Maximum load power generated by a bare tether of given width and lengths versus radius of its circular prograde orbit.

Since eccentricity vanishes throughout, orbit dynamics is just described by the following equation:

$$
\left\langle\left|\dot{W}_{\mathrm{drag}}(a)\right|\right\rangle_{\varphi}=\frac{d}{d t}\left(\frac{\mu_{J} M_{\mathrm{SC}}}{2 a}\right) .
$$

The full magnetic power, involving both drag power on the SC and back power on the corotating and magnetized Jovian plasma $\left(-\bar{F}_{\text {mag }}\right) \cdot \bar{\nu}_{\mathrm{pl}}$, reads

$$
\begin{aligned}
\dot{W}_{\text {mag }} & =\bar{F}_{\text {mag }} \cdot \bar{\nu}^{\prime}=-L \bar{I}_{\text {av }} \cdot \bar{E}_{m}=-L I_{\text {av }} E_{m} \\
\cos \varphi & =\dot{W}_{\text {drag }} \times \nu^{\prime} \nu .
\end{aligned}
$$

As regards load power, one readily finds [3], [7]

$$
\dot{W}_{\text {load }} /\left|\dot{W}_{\text {mag }}\right| \equiv \text { efficiency } \eta=\frac{1-\varsigma}{1-2 \varsigma / 5} .
$$

Load power, which is proportional to $(1-\zeta) \zeta^{3 / 2}$, is maximum at $\zeta=0.6$, yielding $\eta_{\max }=10 / 19$. We then find

$$
\left\langle\dot{W}_{\text {load }}(a)\right\rangle_{\varphi}=\eta(\varsigma) \frac{\nu^{\prime}}{\nu} \frac{d}{d t}\left(\frac{\mu_{J} M_{\mathrm{SC}}}{2 a}\right)
$$

which is depicted for prograde motion in Fig. 9, where (19)-(21) were used. Equation (24) readily yields the energy 


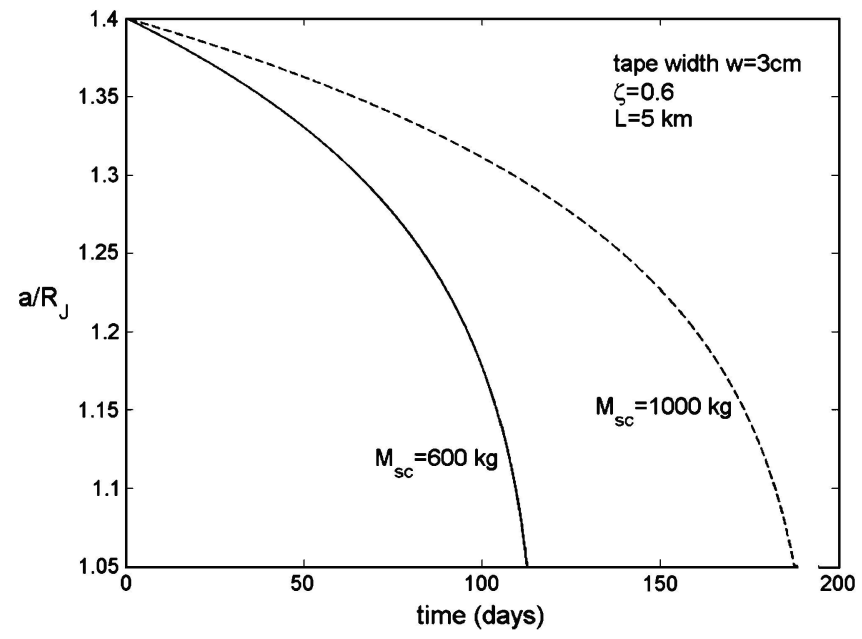

Fig. 10. Second-stage near-circular prograde orbit evolution of SC with masses about three and five times the initial tether mass $m_{t}=202 \mathrm{~kg}$, captured at perijove $1.4 R_{J}$, after cutting to a tenth its length.

generated at the load in deorbiting from some initial to final circular orbits

$$
\begin{aligned}
\frac{W_{\text {load }}}{M_{\mathrm{SC}}} & =-\eta(\varsigma) \frac{\mu_{J}}{2} \int_{a_{i}}^{a_{f}} \frac{d a}{a^{2}}\left[1 \pm\left(\frac{a}{a_{s}}\right)^{3 / 2}\right] \\
& =32.7(14.7) \frac{\mathrm{kWh}}{\mathrm{kg}}
\end{aligned}
$$

for retrograde (prograde) motion, respectively; we finally took values $\zeta=0.6, a_{i}=1.3 R_{J}$, and $a_{f}=1.05 R_{J}$. Fig. 10 shows the $a(t)$ for prograde motion as obtained from (21) for $L=$ $5 \mathrm{~km}$ and $m_{t}=20.3 \mathrm{~kg}$, and two $M_{\mathrm{SC}}$ values, again using the value $\zeta=0.6$ in (20).

\section{SCiEntific Applications}

A basic mission goal would be determining the structure and dynamics of the Jovian atmosphere, which was actually one goal of the JESM too. Space- and time-resolved observations, essential for understanding transport processes, would be possible. Data registered over many months would allow studying atmospheric variability over different time scales. This includes clarifying how and why the stratospheric thermal structure varies with time and tracking the evolution of lightning storms [12]. Measurements so close to Jupiter's surface would also allow increasing our knowledge of its interior. This would include accurate high-resolution determination of gravity and magnetic fields, and determining the bulk abundance of water. Accurate mapping of the gravity field could show nonhydrostatic pressure effects where water was absent. Measuring brightness temperature at millimeter wavelengths at close range would give water abundance without the ambiguity of remote sensing [12].

The radial range 1.3-1.4 $R_{J}$ contains both the inner region of the halo ring and the $2: 1$ Lorentz resonance $\left(\Omega_{\text {orb }}=2 \Omega_{j}\right.$ or $a=$ for $a_{s} / 2^{2 / 3}$ ). The $2: 1$ Lorentz resonance, basically due to a Schmidt coefficient $g_{2}^{2} \sim 0.4-0.5$ Gauss of the associate Legendre function in the spherical harmonic expansion of the

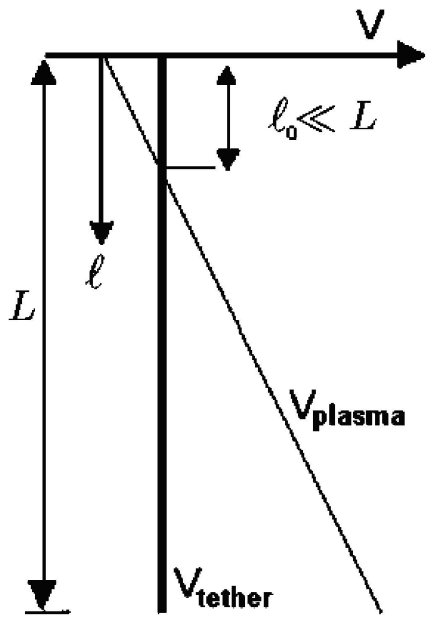

Fig. 11. Schematics of electrically floating bare tether (zero current at both ends) during each half spin period with the hollow cathode at the "wrong" (anodic) end. The anodic segment collecting electrons is a fraction about $\left(m_{e} / m_{i}\right)^{1 / 3}$ of the total length $L$, to allow ion collection over the cathodic negatively biased segment to balance electron collection.

magnetic field, is the strongest by far and is located at about $1.4 R_{J}$. In situ detection of (charged) grains might advance well beyond remote-imaging ring studies [12], [13], allowing in situ measurement of dust charge, mass, velocity, and chemical composition. The Lorentz force on a charged grain results in sensible grain acceleration because of large Jovian magnetic field and orbital velocities and charge-to-mass ratios. Grain-tether interaction makes a complex dusty-plasma problem, involving grain dynamics and charge evolution. Charge equilibrium takes typically longer than grain flight time through different ambient conditions; grain dynamics and charge evolution must be jointly solved. Grains have a typical density of $2 \mathrm{~g} / \mathrm{cm}^{3}$, radius $R_{\mathrm{gr}}$ within a broad range centered at $1 \mu \mathrm{m}$, and charge $Q_{\mathrm{gr}}$ roughly proportional to $R_{\mathrm{gr}}$. As the SC spirals slowly inward, it finds grains acted upon by gravitational $F_{g} \sim R_{\mathrm{gr}}^{3} / a^{2}$, magnetic $F_{\mathrm{mag}} \sim Q_{\mathrm{gr}} \nu_{\mathrm{orb}} B \sim R_{\mathrm{gr}} / a^{7 / 2}$, and electric $F_{\mathrm{el}} \sim Q_{\mathrm{gr}} \nu_{\mathrm{pl}} B \sim R_{\mathrm{gr}} / a^{2}$ forces dependent on size and orbital radius.

Beyond passive measurements, the orbiting bare tether would allow active experiments. During each half spin period with the hollow cathode at the "wrong" (anodic) end, the tether will be electrically floating, with current vanishing at both ends. Because of the large ion-to-electron mass ratio, the motional field $\boldsymbol{E}_{m}$ will bias the tether negatively over most of its length (Fig. 11). Under the impact of attracted ions, the tether will emit secondary electrons at certain yield $\gamma$ and form a beam traveling along magnetic field lines, with particle and energy flux growing with distance $l$ from tether top [14]. The beam electron flux reads

$$
\Phi_{b}(l)=N_{e} \Omega_{e} w \sqrt{\frac{m_{e}}{m_{i}}} \frac{\gamma\left(e E_{t} l\right)}{2 \pi \cos (\text { magnetic dip) }}
$$

which is much weaker than the ambient thermal flux; the beam/ambient density ratio is also very small. The dip in (26) involves the actual multipole-field dip and the angle $\varphi$ of the rotating tether; orbit and spin periods (over $3 \mathrm{~h}$ and, for 


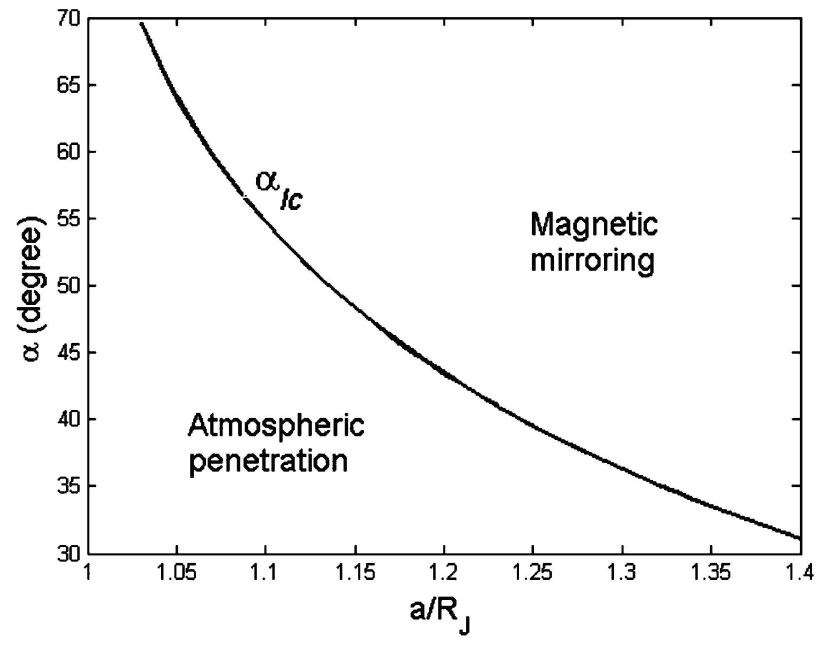

Fig. 12. Loss-cone pitch angle $\alpha_{l c}$ versus $a / R_{J}$ here representing magneticshell parameter.

example, around $10 \mathrm{~min}$, respectively) are reasonably disparate. The pitch-angle $\alpha$ distribution is then [14]

$$
\frac{\Phi_{b}(h, \alpha)}{\Phi_{b}(h)}=\frac{2 / \pi}{\sqrt{\sin ^{2} \alpha-\sin ^{2} \operatorname{dip}(\varphi)}}, \quad-90^{\circ}<\varphi<90^{\circ} .
$$

For the high altitudes of interest, beam electrons with high pitch would bounce back as trapped electrons, whereas lowpitch electrons would imprint auroral lights along a beam track at the upper atmosphere. The pitch range in the beam is $\operatorname{dip}(\varphi)<\alpha<\alpha_{l c}$, where the loss-cone pitch $\alpha_{l c}(a)$ shown in Fig. 12 follows from the no-tilt dipole $B$ model

$$
\sin ^{4} \alpha_{l c}=R_{J}^{6} / a^{5}\left(4 a-3 R_{J}\right) .
$$

While radiation dose is typically the main constraint on the use of tethers at Jupiter, there are other environmental considerations. In particular, surface charging and internal electrostatic discharge (IESD) are possible concerns, which, in particular, might possibly disrupt science. As discussed in previous [15], [16] studies, however, surface charging is normally only a limited concern above the Jovian auroral zone and, to a lesser extent, along the magnetic field lines that connect the auroral zone to Jovian plasma equator. As described in [16], however, these surface potentials are likely to be less than a few kilovolts and are easily controlled (note that they represent a net system potential relative to the plasma and a plasma contactor should suffice to ground them out). IESD [15] is a known and real concern for Jovian missions and is also the result of radiation-high-energy ( $>100 \mathrm{keV}$ ) electrons preferentially penetrate the SC shielding relative to protons and ions and build up charge on isolated conductors or in dielectrics. As reported in [15], the charge collected can build up sufficiently to cause arcs and likely resulted in 42 anomalies on Voyager 1. As in the case of typical radiation effects, shielding can reduce IESD. Limiting isolated conductors and dielectrics and proper grounding were very effective for Galileo at preventing IESD effects. Even so, IESD will need to be considered-while we will not explicitly include it in our study, we note that it can be estimated by computing the charge deposited behind a specified shielding thickness. This can be done by using the simple relationship that electron fluence $\left(\mathrm{e} / \mathrm{cm}^{2}\right)=2.4 \times 107 \bullet \operatorname{dose}(\mathrm{rad}-\mathrm{Si})$. As a rule [15], $10^{10} \mathrm{e} / \mathrm{cm}^{2}$ in less than $10 \mathrm{~h}$ could cause a discharge.

\section{CONCLUSION}

A two-stage mission to place an SC below the Jovian radiation belts, using a spinning bare tether with hollow cathodes at both ends to provide propulsion and power, has been considered. Possible values for tether length and mass are $50 \mathrm{~km}$ and $200 \mathrm{~kg}$, respectively. Lorentz drag on the current induced in the tether would capture and progressively bring an SC, with mass that is three to five times larger, down to a circular (equatorial) orbit below the halo ring. For given capture perijove and tether dimensions, retrograde orbits allow greater SC-to-tether mass ratio for equal accumulated radiation dose, or lower dose for equal mass ratio, but have worse performances regarding thermal and bowing issues. In the second stage, the tether is cut, retaining a segment that is one order of magnitude smaller, which makes the SC to slowly spiral inward over many months while generating large power on board; with single hollow-cathode operation, the tether will electrically float every half spin period. Interesting science opportunities arise for missions below $1.4 R_{J}$, where radiation poses no problem. These include nearby high-resolution observations, over long times, of Jupiter's atmosphere and interior; in situ measurements of charged grains by a dust detector; and auroral sounding of Jupiter's upper atmosphere.

\section{ACKNOWLEDGMENT}

The research described in this paper by H. B. Garrett was carried out at the Jet Propulsion Laboratory, California Institute of Technology, under a contract with the National Aeronautics and Space Administration.

\section{REFERENCES}

[1] "Radioisotope power systems: An imperative for maintaining U.S. leadership in space exploration," Nat. Res. Council, Washington, DC, 2009, Tech. Rep.

[2] Jupiter Europa Orbiter and Jupiter Ganymede Orbiter Concept, last accessed February 2011. [Online]. Available: http://opfm.jpl.nasa.gov/ europajupitersystemmissionejsm/

[3] J. R. Sanmartin, E. C. Lorenzini, and M. Martinez-Sanchez, "Electrodynamic tether applications and constraints," J. Spacecr. Rockets, vol. 47, no. 3, pp. 442-456, 2010.

[4] J. R. Sanmartin, M. Charro, E. C. Lorenzini, H. B. Garrett, C. Bombardelli, and C. Bramanti, "Electrodynamic tether at Jupiter-I: Capture operation and constraints," IEEE Trans. Plasma Sci., vol. 36, no. 5 , pp. 2450-2458, Oct. 2008.

[5] J. R. Sanmartin, M. Charro, E. C. Lorenzini, H. B. Garrett, C. Bombardelli, and C. Bramanti, "Electrodynamic tether at Jupiter-II: Fast moon tour after capture," IEEE Trans. Plasma Sci., vol. 37, no. 4, pp. 620-626, Apr. 2009.

[6] C. Bombardelli, E. C. Lorenzini, and J. R. Sanmartin, "Jupiter power generation at constant orbital energy," J. Propuls. Power, vol. 5, pp. 415-424, 2009.

[7] J. R. Sanmartin, M. Martinez-Sanchez, and E. Ahedo, "Bare wire anodes for electrodynamic tethers," J. Propuls. Power, vol. 9, pp. 353-360, 1993.

[8] A. M. Jablonski, H. G. James, G. Tyc, F. R. Vigneron, C. K. Carrington, C. C. Rupp, and J. K. Harrison, "The BOLAS ionospheric tether mission," in Proc. 49th IAF Congr., Small Satellite Missions Symp., Melbourne, Australia, Sep. 28-Oct. 2, 1998. 
[9] R. P. Hoyt, J. T. Slostad, and S. S. Frank, "A modular momentumexchange/electrodynamic-reboost tether system architecture," presented at the 39th AIAA/ASME/SAE/ASEE Joint Propulsion Conf., Huntsville, AL, Jul. 20-23, 2003, Paper AIAA-2003-5214.

[10] N. Divine and H. B. Garrett, "Charged particle distributions in Jupiter's magnetosphere," J. Geophys. Res., vol. 88, no. A9, pp. 6889-6903, 1983.

[11] H. B. Garrett, I. Jun, J. M. Ratliff, R. W. Evans, G. A. Clough, and R. W. McEntire, Galileo Interim Radiation Electron Model. Pasadena, CA: Jet Propuls. Lab., 2003.

[12] F. Bagenal, T. Dowling, and W. McKinnon, Eds., Jupiter, the Planet, Satellite, and Magnetosphere. Cambridge, U.K.: Cambridge Univ. Press, 2004

[13] J. A. Burns, L. E. Schaffer, R. J. Greenberg, and M. R. Showalter, "Lorentz resonances and the structure of the Jovian ring," Nature, vol. 316, no. 6024, pp. 115-119, Jul. 1985.

[14] J. R. Sanmartin, M. Charro, J. Peláez, I. Tinao, S. Elaskar, A. Hilgers, and M. Martinez-Sanchez, "Floating bare tether as upper atmosphere probe," J. Geophys. Res., vol. 111, no. A11, p. A11310, 2006.

[15] P. Leung, A. C. Whittlesey, H. B. Garrett, P. A. Robinson, Jr., and T. N. Divine, "Environment-induced electrostatic discharges as the cause of Voyager 1 power-on resets," J. Spacecr. Rockets, vol. 23, no. 3, pp. 323330, 1986.

[16] H. B. Garrett, R. W. Evans, A. C. Whittlesey, I. Katz, and I. Jun, "Modeling of the Jovian auroral environment and its effects on spacecraft charging," IEEE Trans. Plasma Sci., vol. 36, no. 5, pp. 2440-2449, Oct. 2008 